\title{
Multi-target device-free tracking using radio frequency tomography
}

\author{
Santosh Nannuru ${ }^{\# 1}$, Yunpeng Li ${ }^{* 2}$, Mark Coates ${ }^{* 3}$, Bo Yang *4 \\ \# Dept. of Electrical and Computer Engineering, McGill University \\ Montreal, Quebec, Canada \\ ${ }^{1}$ santosh.nannuru@mail.mcgill.ca, ${ }^{3}$ mark.coates@mcgill.ca \\ * Multimedia Technology Center, Beijing Univ. of Posts and Telecom. \\ Beijing, China \\ ${ }^{2}$ liyp@bupt. edu.cn, ${ }^{4}$ boyang@bupt . edu.cn
}

\begin{abstract}
This paper examines device-free tracking of multiple targets based on radio-frequency received signal strength (RSS) measurements recorded by a sensor network. We report experimental results that validate, for relatively uncluttered outdoor environments, a recently proposed model in which targets cause additive attenuation. Using this model, we examine the performance of three multi-target tracking algorithms using a experimental sensor network testbed consisting of 24 nodes that conducts surveillance of an outdoor area of size $50 \mathrm{~m}^{2}$. The experiments are restricted to the case of a fixed number of targets (up to four). For four targets, all algorithms are able to track with average error less than $1 \mathrm{~m}$ (as measured using the secondorder OMAT metric); for two targets the error is close to $0.2 \mathrm{~m}$.
\end{abstract}

\section{INTRODUCTION}

We address the task of tracking multiple mobile targets in a "device-free" setting, where targets do not carry active or passive devices to assist the tracking system. This form of tracking has important applications in military surveillance, search-and-rescue operations, through the wall imaging, and healthcare environments [1], [2]. Wireless sensor networks that perform tracking based on radio-frequency (RF) received signal strength (RSS) measurements are attractive because they are relatively inexpensive and can be deployed quickly. The RF measurement modality has the advantages, compared to alternatives such as infrared and video, of having the potential to penetrate walls and other non-metallic obstacles.

The imaging of an area to detect mobile targets based on the additional attenuation and fluctuations they cause in wireless transmissions has been named "radio-frequency (RF) tomography" [3]. In previous work, tracking using radiofrequency tomography principles has been primarily limited to a single target [3], [4], [5], [6]. Recently, Thouin et al. developed a multiple target tracking algorithm for the RF tomography setting [7]. The paper proposes a measurement model that assumes that the attenuation caused by individual targets combines in an additive fashion in the multi-target setting. The performance assessment in [7] was based entirely on simulation; no practical experimentation was conducted.

This paper reports on an experimental study to validate the proposed additive model and to assess the practical tracking capabilities of the proposed system. We restrict our attention to relatively uncluttered outdoor environments. In this setting, our experiments confirm that the model is acceptably accurate, although the variance in the measurements is relatively high. For the case where there is a fixed and known number of targets, we examine the performance of three candidate multitarget tracking algorithms: the bootstrap particle filter [8], the multiple particle filter [9], [10], and a Markov Chain Monte Carlo (MCMC) filter [11], [7]. Our experiments are conducted using a experimental sensor network testbed consisting of 24 nodes deployed in an uncluttered outdoor area of size $50 \mathrm{~m}^{2}$. The experiments are restricted to the case of a fixed number of targets (up to four), and illustrate that in this setting, accurate real-time tracking can be performed.

The rest of the paper is organized as follows. In the following subsection, we discuss related work in more detail. Section II provides a detailed problem statement and describes the measurement model adopted for the multi-target case. It also presents the results of experiments conducted to validate this model. In Section III we briefly review the multi-target tracking algorithms we have employed. The sensor-network experimental setup and tracking results are discussed in IV. Concluding remarks are presented in Section V.

\section{A. Related Work}

There has been much recent work addressing device-free localization of targets using wireless sensor networks, with the review paper [2] providing a good overview of the many techniques. The problem is related to MIMO radar [12] and classical multistatic radar tracking, but the underlying measurement systems and signal models are very different.

Zhang et al. were among the first to develop tracking systems based on the interference caused by moving objects [13], [14], [15]. These systems achieve good tracking performance, but the employed models are developed specifically for ceiling-mounted sensor nodes and the spacing between sensor nodes needs to be relatively small (2-4m). Calibration is also required, and this is not practical in important scenarios we wish to consider (search-and-rescue, military surveillance). 
Of the techniques that have directly addressed tracking and incorporated basic models of target dynamics, the majority have focused on single target tracking. Wilson and Patwari proposed a method in [3], [4] that first applies an inverse imaging algorithm to obtain an attenuation map and then applies a Kalman filter to track the peak in the map. In [5], $\mathrm{Li}$ et al. introduced a sequential Monte Carlo algorithm that incorporates online Expectation-Maximization to train model parameters. The approach was extended to incorporate simultaneous localization of the sensor nodes in [6].

As discussed above, Thouin et al. focused on the multitarget tracking problem in [7], proposing a measurement model that assumed that targets caused additive attenuation effects. They introduced a new moment-based filtering algorithm and reported simulation results showing the ability to track accurately and efficiently.

\section{Models and Problem Statement}

\section{A. Problem statement}

When a sensor network communicates through transmission of wireless packets, the received signal strength (RSS) measurements on a link connecting sensor nodes is affected by the distance between the sensors and the interference caused by static and moving objects. When a target moves through the sensed region, it affects different links, and changes in RSS values can be used to perform tracking.

Our experimental system is a wireless sensor network of $R$ nodes and $M=\frac{R^{2}-R}{2}$ bidirectional links. Each node successively broadcasts packets at short time intervals $(8.3 \mathrm{~ms}$ in our testbed) and the other nodes measure the RSS. A single measurement interval corresponds to the period required for all nodes to transmit (200ms in our 24-node testbed). The measurements are then the RSS values of all bidirectional links. For link $i$ and time step $k$, we denote this RSS value $\gamma_{i}(k)$; it is the average of the RSS values recorded on the forward and reverse links. We stack the RSS measurements into a vector $\gamma(k)$.

The RSS model adopted in this paper was proposed in [7] and is an extension of the empirical single target model of [6]. Under this model, the RSS value $\gamma_{i}(k)$ is split into three main terms: $\gamma_{i}(k)=\bar{\gamma}_{i}+y_{k}^{i}+\zeta_{k}^{i}$. Here $\bar{\gamma}_{i}$ is the average RSS on link $i$ when no target is present, $y_{k}^{i}$ is the attenuation on link $i$ at time step $k$ due to mobile targets, and $\zeta_{k}^{i}$ is additive white Gaussian noise affecting the measured RSS on link $i$ at time step $k$. We assume that there is a time period during which we can gather measurements on all links when no target is present in order to estimate $\bar{\gamma}_{i}$. The measurements we use for tracking at time step $k$ are then $z_{k}=\gamma_{k}-\bar{\gamma}$.

We address the problem of tracking a known and fixed number of targets. The $n^{t h}$ target is identified at time $k$ by by its state $x_{k, n}$. We assume that the motion of each target is specified by a Markovian dynamic model $f\left(x_{k} \mid x_{k-1}\right)$. If $N_{k}$ targets are present in the sensed region, then the combined state is $X_{k}=\left[x_{k, 1}, x_{k, 2}, \ldots, x_{k, N_{k}}\right]$. Our goal is to estimate the posterior distribution at every time $k$ of the $N_{k}$ targets given all the measurements up to time $k$ (marginalized with respect to time). Thus at every time step $k$ we want to approximate the distribution $p\left(X_{k} \mid z^{(1: k)}\right)$ where $z^{(1: k)}$ represents all the observations up to time $k$, i.e., $z^{(1: k)}=\left\{z_{1}, z_{2}, \cdots, z_{k}\right\}$. Since the state posterior distribution has all the information about the target states and associated uncertainty, we can derive point estimates from the approximation.

In this paper, we adopt a very simple random walk model for the target motion. Our main motivation for the simple model is to allow for very general movements (humans walking, stopping, turning). The state evolution equation we adopt is:

$$
x_{k+1, n}=x_{k, n}+\sigma_{v} V_{k}
$$

where $V_{k}$ is distributed according to a Gaussian distribution $\mathcal{N}\left(0, I_{2 \times 2}\right)$. The targets are assumed to move independently of each other. In this work we assume that $\sigma_{v}$ is a known constant, but online EM procedures [5] could be incorporated to jointly track the targets and estimate this value.

\section{B. Measurement model}

Li et al. proposed a single target measurement model for RF tomography in an uncluttered outdoor region based on experimental studies [5]. The RSS attenuation on link $j$ caused by a target at position $x$ is modeled as

$$
g_{j}(x)=\phi \exp \left(-\frac{\lambda_{j}(x)}{\sigma_{\lambda}}\right)
$$

where $\phi$ and $\sigma_{\lambda}$ are attenuation parameters based on physical properties of the targets. The value $\lambda_{j}(x)$ is

$$
\lambda_{j}(x)=d_{1}(x)+d_{2}(x)-d_{12}
$$

where $d_{1}(x)$ and $d_{2}(x)$ are distances between target at location $x$ and the two sensors forming the link, $d_{12}$ is the distance between the sensors. $\lambda_{j}(x)$ captures a notion of the distance between the target and the line-of-sight link between transmitter and receiver. In this model, when the target at $x$ is close to link $j, \lambda_{j}(x)$ is large, and hence the attenuation $g_{j}(x)$ is small.

Thouin et al. proposed a multi-target extension of this model in [7]. The extended model assumes that the attenuation caused by the presence of multiple targets is equal to the sum of the attenuation due to each of the targets present alone. Thus if $g_{j}\left(x_{n}\right)$ is the attenuation on link $j$ due to the $n^{t h}$ target located at $x_{n}$, then the total attenuation on link $j$ due to all of the targets combined is

$$
g_{j}\left(X_{k}\right)=\sum_{n=1}^{N_{k}} g_{j}\left(x_{k, n}\right)
$$

where $N_{k}$ is the total number of targets within the field of observation. This being the model-predicted attenuation, the observed noisy attenuation values at time step $k$ is given by

$$
z_{k}=g\left(X_{k}\right)+\sigma_{z} s_{k}
$$

where $g=\left[g_{1}, g_{2}, \cdots, g_{M}\right], M$ is the number of links, and $s_{k}$ is noise, assumed to be distributed according to $\mathcal{N}\left(0, I_{M \times M}\right)$. We assume that $\sigma_{z}$ is known or estimated during the period when background attenuation values are learned. 


\section{Experimental Validation of Additive Model}

We assess the validity of the additive attenuation model using single-link sensor measurements when multiple targets affect the link. Our experiments are conducted for a link with the sensors separated by 8 meters. The transceivers of the sensor nodes are system-on-chip (SoC) TI CC2530 devices; each node has a monopole antenna and uses the $2.4 \mathrm{GHz}$ IEEE 802.15.4 standard for communication. Figure 1 depicts the experimental setup. In each experiment, Target A stands on the line-of-sight path between the sensor nodes (the positions are labeled "LOS marker" in Figure 1). Target B walks along a trajectory perpendicular to the line-of-sight path, crossing close to the position of Target A. For each experiment, Target B completes 10 crossings and approximately 22,000 RSS measurements are measured by the receiving sensors. We also conduct similar experiments when only Target A or Target B is present.

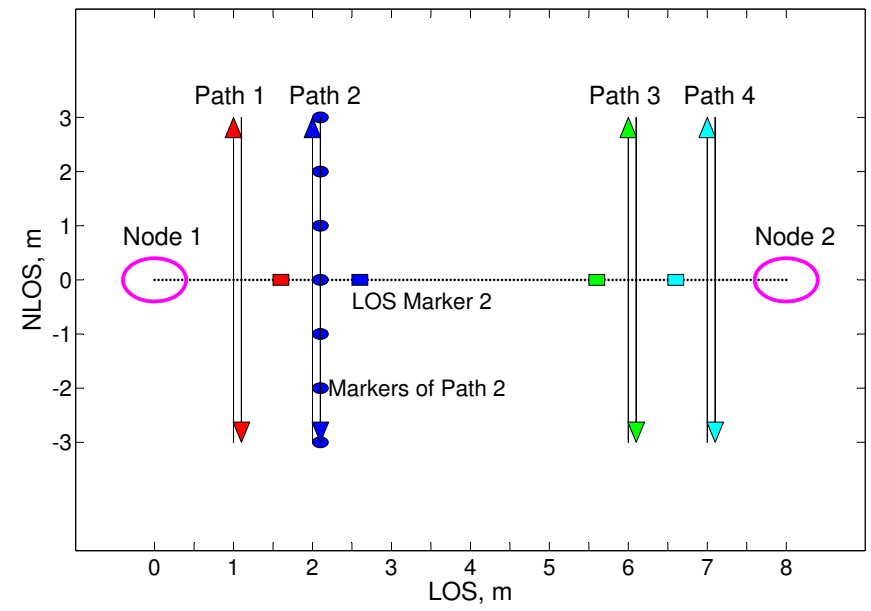

Fig. 1. The figure illustrates the single link experiment setup. Markers on LOS indicate different positions of stationary Target A. Arrows indicate path of moving Target B.

Figure 2 presents a scatter plot of the attenuation values after the background RSS estimates have been subtracted. This figure also shows the average attenuations when Target $\mathrm{A}$ is alone (dashed line) and when Target B is alone (blue line with square markers). We compare the average attenuation of the two targets with the model in (5). In the model we set $\phi$ for each target to the mean attenuation value when the target is alone and choose $\sigma_{\lambda}=0.04$, which has been observed to be a good fit for the outdoor environment [6]. The additive model provides a good explanation of the experimental average attenuation, particularly in the region of interest ( $\operatorname{small} \lambda$ ).

Figure 3 depicts a histogram of the differences between the attenuation values predicted by the model (4) and the measured values. These differences should be explained by the additive Gaussian noise in (5). Superimposed is the best-fit Gaussian model. The Gaussian fit has mean $-0.029 d B$ and standard deviation is $2.25 d B$. The fact that the mean is close to zero is encouraging, but the histogram does not correspond to a Gaussian distribution. The quantile-quantile plot in Figure 3 shows

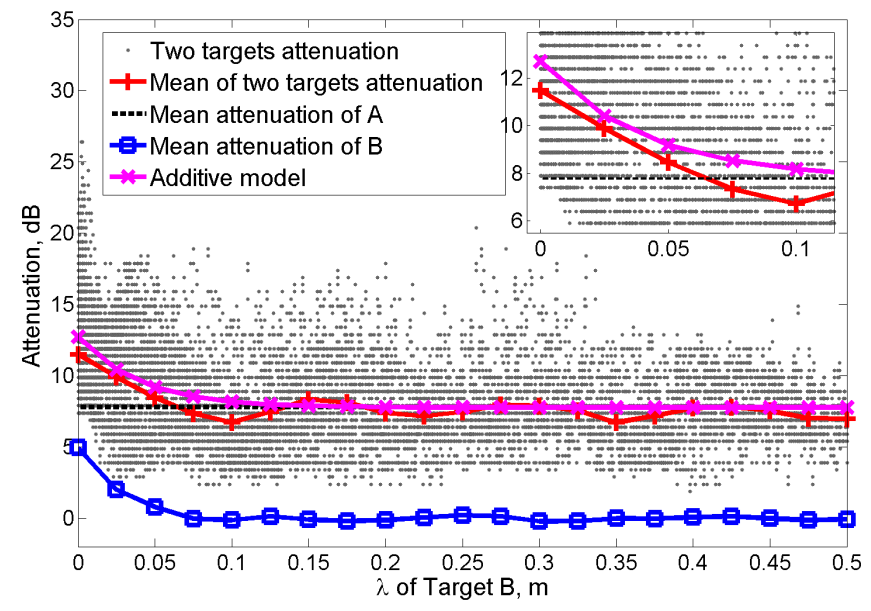

Fig. 2. Signal attenuation levels versus parameter $\lambda$ for the single target and two targets case. Both experimental and model predicted attenuation levels are plotted when two targets are present.
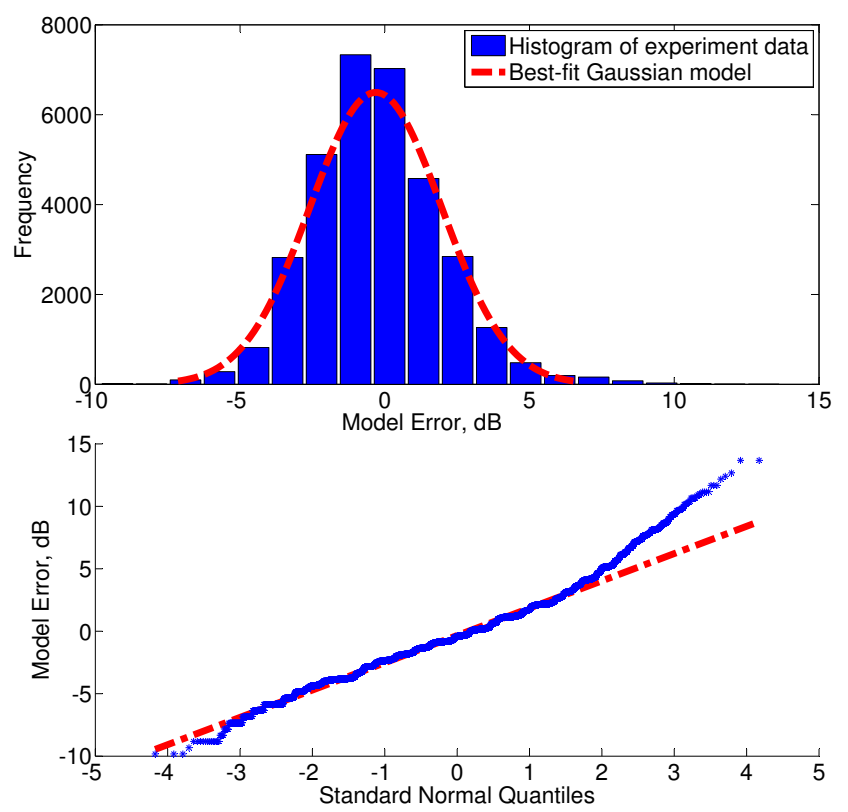

Fig. 3. Validation of the error as Gaussian model. Top figure shows a histogram of the model error and the corresponding best-fit Gaussian. The bottom figure shows a quantile-quantile plot of the model error.

significant discrepancy between the sample quantiles and the standard normal quantiles. There are multiple measurements where the observed attenuation is much higher than predicted by the model; the empirical distribution is skewed to the right and has a relatively heavy tail. The poor match is confirmed by a Lilliefors test, Anderson-Darling test, and D'Agostino test, which all reject the null hypothesis that the differences are normally distributed (with significance 0.05).

Despite the poor fit of the Gaussian, we observe that using the Gaussian model still leads to reasonably good tracking performance (see Section IV). It simplifies the computations and since there are many hundreds of links it is important that log-likelihoods can be evaluated relatively quickly. 


\section{MUlTi TARGET TRACKING ALGORITHMS}

In this section we briefly review the three particle filtering algorithms we employ for multi-target tracking. The first is a simple implementation of the bootstrap particle filter [8] $(\mathrm{BPF})$. It is based on the principle of sequential importance resampling. At every time step, the BPF maintains a weighted set of particles to approximate the posterior distribution. Resampling is performed periodically to ensure that the particle set remains sufficiently diverse.

The bootstrap particle filter is known to struggle when faced with high-dimensional problems. To address this limitation, Djuric et al. introduced the multiple particle filter (MPF) in [9], [10]. They suggested using one low-dimensional particle filter for each of the targets being tracked. The multiple particle filter is summarized in Algorithm 1.

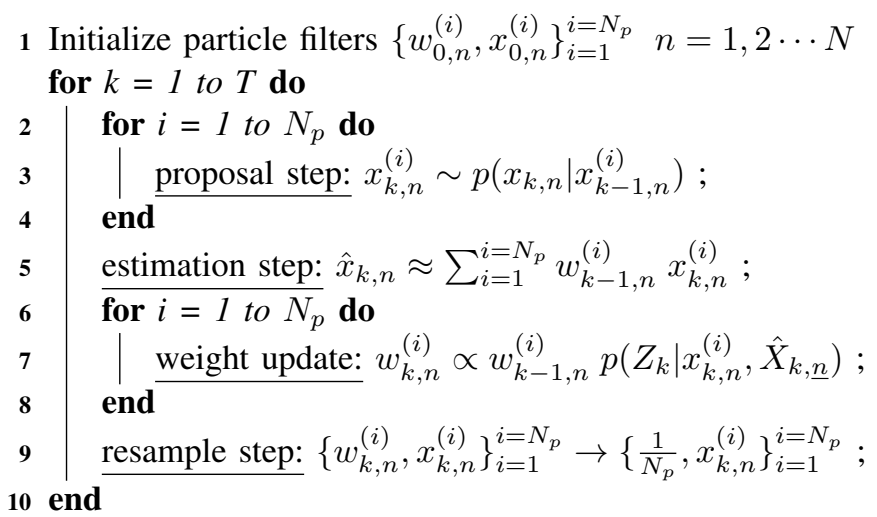

Algorithm 1: Multiple Particle Filter

The MPF maintains a separate particle filter for each of the $N$ targets; $N_{p}$ is the number of particles per target. In our implementation, each filter is a bootstrap particle filter. The weight update step for the individual filters cannot be performed independently of the other target states because the computation of the measurement likelihood requires the combined state information. Thus for the particle filter corresponding to $n^{\text {th }}$ target an estimate of the other target states $\hat{X}_{k, \underline{n}}=\left[\hat{x}_{k, 1} \ldots \hat{x}_{k, n-1}, \hat{x}_{k, n+1} \ldots \hat{x}_{k, N}\right]$ is obtained. The target state estimates $\hat{x}_{k, n}$ are calculated as a weighted average of the current particles $\left\{x_{k, n}^{(i)}\right\}$ using the weights from the previous time step $\left\{w_{k-1, n}^{(i)}\right\}$.

The third particle based method is a Markov Chain Monte Carlo (MCMC) filter. It constructs a Markov chain which has the desired marginal posterior as its stationary distribution. We use a modified version of the filter originally described in [11] (see [7] for details). The pseudocode for the MCMC filter is presented in Algorithm 2. The MCMC chain is initialized by choosing the particle $X_{k}^{(0)}$ from the previous time step that has the highest likelihood for the current observation and we set $X_{k-1}^{(0)}=X_{k}^{(0)}$. The acceptance probabilities $\rho_{1}$ and $\rho_{2}$ are calculated so that the samples are distributed according to the required posterior distributions. In practice, a good sample set is obtained by ignoring the initial $N_{\text {burn }}$ samples and then selecting one in every $N_{\text {thin }}$ samples from the sequence.

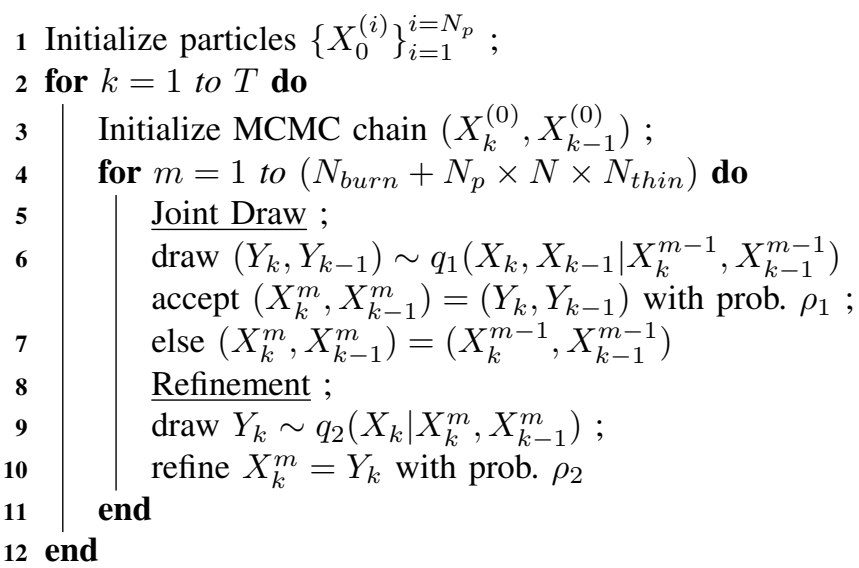

Algorithm 2: MCMC Filter

\section{EXPERIMENTS AND RESULTS}

\section{A. Experiment setup}

Our experiment are conducted using a sensor network comprised of 24 wireless sensor nodes, deployed in a $7 \mathrm{~m} \times 7 \mathrm{~m}$ square layout in an empty grass field (see Figure 4). The transceivers of the sensor nodes are system-on-chip (SoC) TI CC2530 devices; each node has a monopole antenna and uses the $2.4 \mathrm{GHz}$ IEEE 802.15.4 standard for communication.

A simple token ring protocol is used to control transmission. During each time interval of $8.3 \mathrm{~ms}$, one node broadcasts a data packet. All of the other nodes receive this packet and measure the RSS. The token is then passed to the next node. A complete set of measurements, corresponding to a transmission from each of the 24 sensors, is thus collected every $200 \mathrm{~ms}$. This constitutes a time step for the algorithms.

We perform three different experiments, repeating each ten times. In the first experiment two targets move inside the sensor network along a square trajectory. The targets start from diagonally opposite corners of the square and move in anti-clockwise direction. In second experiment two targets move along a zigzag trajectory (see Figure 4). In the third experiment, four targets are present. Two targets move around a bigger square in an anti-clockwise direction, starting in opposite corners. The other two other targets move around a smaller square in a clockwise direction (see Figure 5).

All of the targets are present for the entire duration of the experiment. Two rounds of the trajectory are completed by each target in experiments one and two. Two rounds of the outer square and four rounds of the inner square are completed by the targets in experiment three. Visible markers are placed along the target trajectories and we record the times when targets cross the marker locations to establish a ground-truth trajectory.

\section{B. Tracking performance}

The measurement model parameters are set to be $\phi=5$, $\sigma_{z}=1, \sigma_{\lambda}=0.04$. These values are selected because they are the average values observed in many outdoor experiments. Human targets have caused average line-of-sight attenuations 
ranging from $3-8 \mathrm{~dB}$; the value of $\phi=5$ is an adequate approximation. The tracking performance is relatively robust to changes of these parameter values. The target motion model parameter is set to $\sigma_{v}=0.2$; this parameter effectively places a bound on the distance a target is likely to travel in $200 \mathrm{~ms}$ and the value 0.2 reflects that we are trying to track walking targets.

In the algorithms, we vary the number of particles per target over the range $N_{p}=50,250,500$. For the MCMC algorithm, the burn-in is $N_{\text {burn }}=1000$ and the thinning factor is $N_{\text {thin }}=3$. For all of the filters, the initial set of particles for each target is independently drawn from a Gaussian distribution of unit variance, centered around the actual target location. The estimated target locations are obtained by computing the weighted average of the particles.

To compare and evaluate the algorithms, we need an error metric to measure the proximity of the estimated and the ground truth tracks. We use the OSPA metric [16], which for a fixed number of targets reduces to the $p$-th order OMAT metric:

$$
d_{p}(X, Y)=\left(\frac{1}{n} \min _{\pi \in \Pi} \sum_{i=1}^{n} d\left(x_{i}, y_{\pi(i)}\right)^{p}\right)^{1 / p}
$$

where $\Pi$ is the set of possible permutations of $\{1,2, \ldots, n\}$, $d(x, y)$ is the Euclidean distance between $x$ and $y, X=$ $\left\{x_{1}, \ldots, x_{n}\right\}$ and $Y=\left\{y_{1}, \ldots, y_{n}\right\}$ are arbitrary sets and $p$ is a fixed parameter. We use the value $p=2$. The OMAT error is then equivalent to the root mean square error under the best possible association between estimated target positions and ground truth. The track estimates are obtained by connecting over time the best possible association at every time step.

\begin{tabular}{|c|c|c|c|c|c|c|c|}
\hline & & \multicolumn{2}{|c|}{ BPF } & \multicolumn{2}{c|}{ MPF } & \multicolumn{2}{c|}{ MCMC } \\
\cline { 3 - 8 } & & Err. & Time & Err. & Time & Err. & Time \\
\hline Exp. & $N_{p}$ & $(\mathrm{~m})$ & $(\mathrm{ms})$ &.$(\mathrm{m})$ & $(\mathrm{ms})$ & $(\mathrm{m})$ & $(\mathrm{ms})$ \\
\hline \multirow{3}{*}{ Exp. 1 } & 50 & 0.42 & 20 & 0.20 & 17 & 0.23 & 1008 \\
\cline { 2 - 8 } & 250 & 0.21 & 101 & 0.20 & 88 & 0.20 & 1978 \\
\cline { 2 - 8 } & 500 & 0.20 & 217 & 0.20 & 179 & 0.20 & 3374 \\
\hline \hline \multirow{2}{*}{ Exp. 2 } & 50 & 0.64 & 19 & 0.23 & 17 & 0.22 & 973 \\
\cline { 2 - 8 } & 250 & 0.23 & 95 & 0.22 & 84 & 0.22 & 1889 \\
\cline { 2 - 8 } & 500 & 0.27 & 202 & 0.22 & 170 & 0.22 & 3137 \\
\hline \multirow{2}{*}{ Exp. 3 } & 50 & 1.34 & 62 & 0.96 & 48 & 0.77 & 1709 \\
\cline { 2 - 8 } & 250 & 1.06 & 338 & 0.90 & 244 & 0.69 & 4333 \\
\cline { 2 - 8 } & 500 & 0.88 & 803 & 0.91 & 493 & 0.63 & 7636 \\
\hline
\end{tabular}

TABLE I

AVERAGE ERROR (IN METER) AND AVERAGE COMPUTATIONAL TIME (IN MILLISECONDS) PER TIME STEP FOR DIFFERENT EXPERIMENTS USING DIFFERENT ALGORITHMS WITH $N_{p}=50,250 \& 500$.

We run each algorithm with 10 different random initializations. Since each experiment is repeated 10 times, we compute an average error (over all the 100 track-initialization pairs). Table I presents the errors for the cases $N_{p}=50,250$, and 500 . The table also shows the average computation time required per time step. All the processing was conducted in Matlab running on computers with the same configuration (2 Xeon 4-core $2.5 \mathrm{GHz}$ processors, 14GB RAM). Computational time is measured using the tic, toc routines in Matlab.

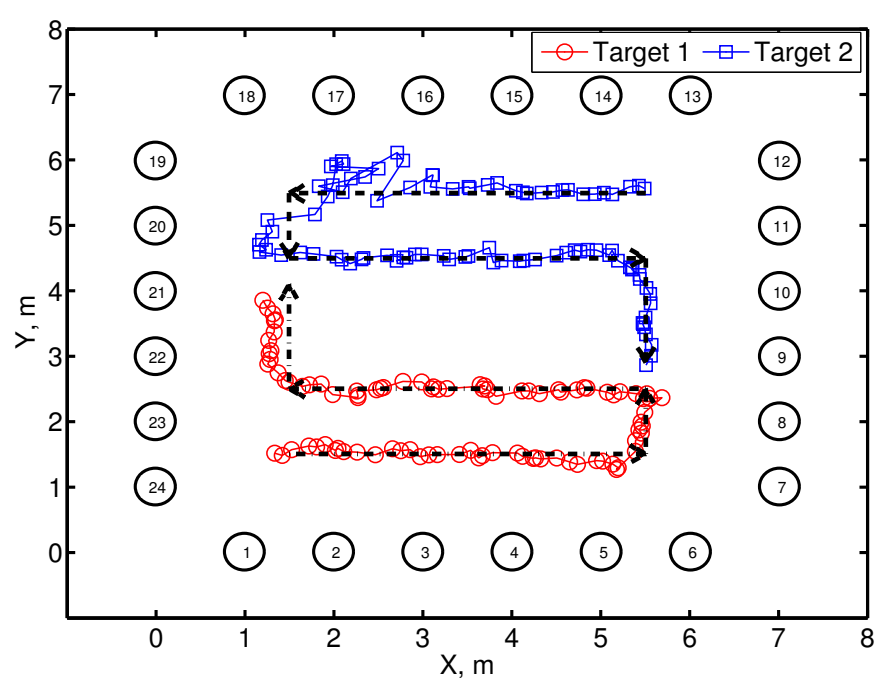

Fig. 4. A network of 24 sensors (indicated as numbered circles) deployed in a square layout and having $M=276$ communication links. Also shown in the figure is the true trajectory when two targets are moving in a zigzag path (indicated by dotted arrows) for the second experiment and the estimated target tracks obtained using the MPF algorithm with $N_{p}=500$.

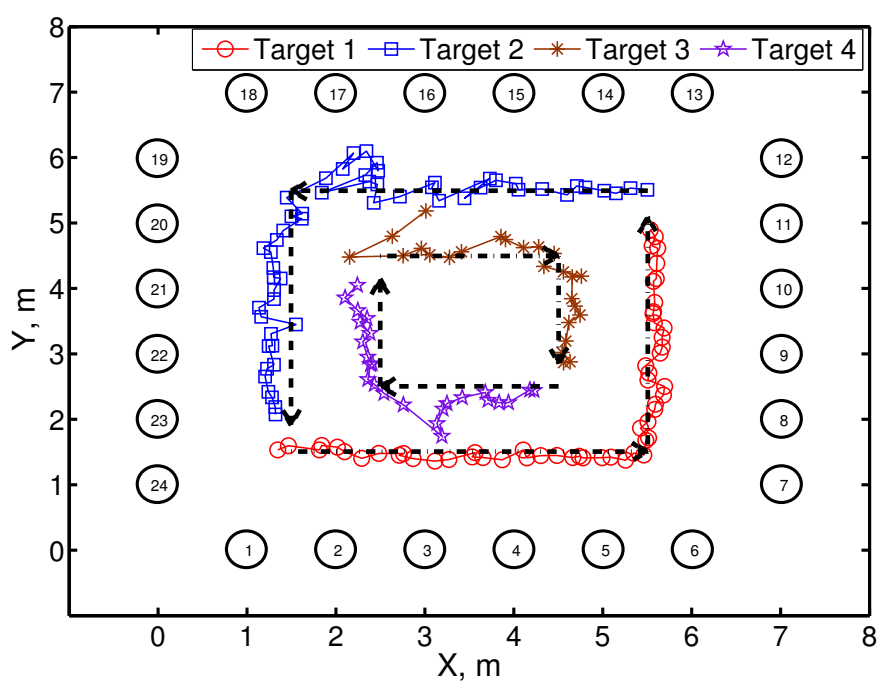

Fig. 5. The figure shows ground truth trajectory (indicated by dotted arrows) for the third experiment with four targets and the estimated target tracks obtained using the MCMC algorithm with $N_{p}=500$.

The results illustrate that the MPF requires only 50 particles per target to track effectively and that it can execute in realtime (computational time is less than the $200 \mathrm{~ms}$ measurement interval). It significantly outperforms the BPF, which needs many more particles to achieve comparable accuracy. For four targets, the MCMC filter is more accurate than the MPF, but it is almost impossible to execute as a real-time system. The results for the four-target experiment do illustrate that there is room for improvement over the MPF algorithm. Interestingly, the accuracy of the MPF does not improve as the number of 

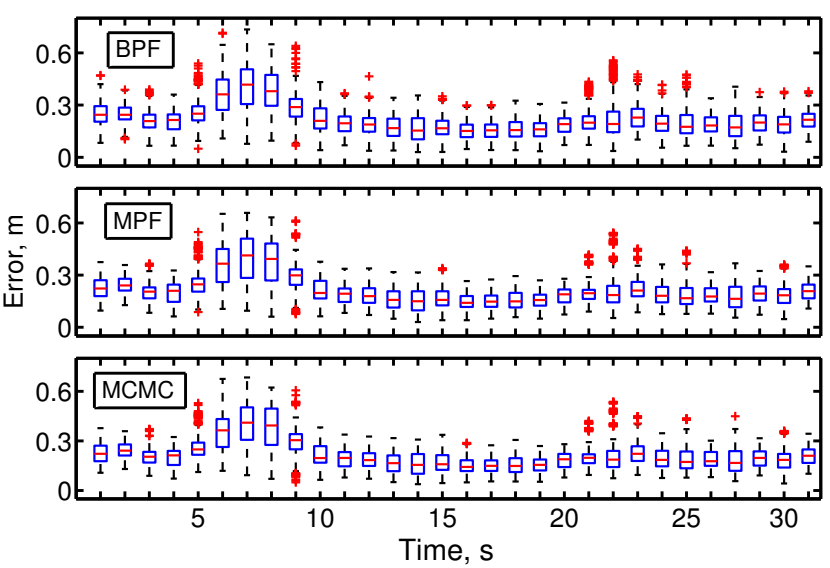

(a) Box and whisker plot of error for experiment 2
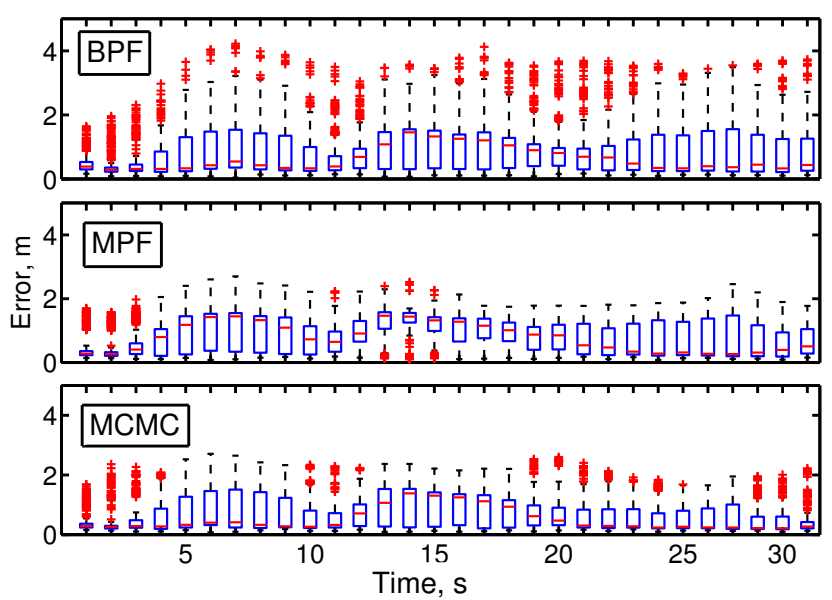

(b) Box and whisker plot of error for experiment 3

Fig. 6. Box and whisker plot of the error for experiments $2 \& 3$ as simulation progresses for the algorithms BPF, MPF and MCMC with $N_{p}=500$. The box ranges from $25^{t h}$ to $75^{t h}$ percentile and the line within the box indicates the median value and the pluses indicate outliers.

particles is increased, indicating that the extra error is probably due to the approximation employed in the algorithm during the weight update stage.

Sample tracking trajectories are shown in Figure 4 (two targets, MPF algorithm) and Figure 5 (four targets, MCMC filter). The tracking is generally accurate, with the occasional departure from the true trajectory. Figures 6(a) and 6(b) show box and whisker plots of error as a function of time for the different algorithms. The BPF has numerous outliers indicating frequent tracking errors. The MPF and MCMC filter have fewer outliers and the worst-case errors are on the order of $0.6 \mathrm{~m}$ for the two-target case and $2.5 \mathrm{~m}$ for the four-target case.

\section{CONCLUSION}

This paper has provided some experimental verification of an additive attenuation measurement model for multitarget RF tomography. The experiments indicated that the model adequately captures the average attenuation; the noise, although close to zero-mean, is not well described by the
Gaussian model. We examined the performance of three multitarget tracking algorithms using data collected using a 24-node sensor network testbed deployed over $50 \mathrm{~m}^{2}$ in an open field. The results indicated that the multiple particle filter of Djuric et al. [9] can perform real-time tracking with relatively good accuracy. In future work we will conduct a more thorough assessment of the accuracy of the model and examine more complicated tracking scenarios such as interacting targets and a variable number of targets.

\section{ACKNOWLEDGMENT}

This research was supported by the National Science and Engineering Research Council of Canada and industrial partners through the hSITE Research Network. The authors thank Yan Zeng for her help in conducting the single link experiments. We also thank Xue Liu and Guang Zhao for conducting experiments at Beijing University of Posts and Telecommunications.

\section{REFERENCES}

[1] M. Moussa and M. Youssef, "Smart devices for smart environments: device-free passive detection in real environments," in Proc. Int. Conf. Perv. Comp. and Comm., Galveston, TX, U.S.A., Mar. 2009.

[2] N. Patwari and J. Wilson, "RF sensor networks for device-free localization: measurements, models, and algorithms," Proc. IEEE, vol. 98, no. 11, pp. 1961-1973, Nov. 2010.

[3] J. Wilson and N. Patwari, "Radio tomographic imaging with wireless networks," IEEE Trans. Mobile Computing, vol. 9, no. 5, pp. 621-632, Jan. 2010.

[4] — , "See through walls: motion tracking using variance-based radio tomography networks," IEEE Trans. Mobile Computing, vol. 10, no. 5, pp. 612-621, May. 2011.

[5] Y. Li, X. Chen, M. Coates, and B. Yang, "Sequential Monte Carlo radio-frequency tomographic tracking," in Proc. Int. Conf. on Acoustics, Speech and Signal Processing, Prague, May 2011.

[6] X. Chen, A. Edelstein, Y. Li, M. Coates, M. Rabbat, and A. Men, "Sequential Monte Carlo for simultaneous passive device-free tracking and sensor localization using received signal strength measurements," in Int. Conf. on Information Processing in Sensor Networks, Chicago, U.S.A., April 2011.

[7] F. Thouin, S. Nannuru, and M. Coates, "Multi-target tracking for measurement models with additive contributions," in Int. Conf. on Information Fusion, Chicago, U.S.A., July 2011.

[8] N. Gordon, D. Salmond, and A. Smith, "Novel approach to nonlinear/non-Gaussian Bayesian state estimation," Radar and Signal Processing, IEE Proceedings F, vol. 140, no. 2, pp. 107-113, Apr. 1993.

[9] P. Djuric, T. Lu, and M. Bugallo, "Multiple particle filtering," in IEEE Int. Conf. Acoustics, Speech and Signal Proc., Honolulu, HW, USA, June 2007.

[10] M. Bugallo, T. Lu, and P. Djuric, "Target tracking by multiple particle filtering," in Proc. IEEE Aerospace Conf., Big Sky, MT, March 2007.

[11] S. K. Pang, J. Li, and S. Godsill, "Models and algorithms for detection and tracking of coordinated groups," in Proc. IEEE Aerospace Conf., Big Sky, MT, Mar. 2008.

[12] A. Haimovich, R. Blum, and L. Cimini, "MIMO radar with widely separated antennas," IEEE Signal Processing Magazine, vol. 25, no. 1, pp. 116-129, Jan. 2008.

[13] D. Zhang, J. Ma, Q. Chen, and L. Ni, "An RF-based system for tracking transceiver-free objects," in Proc. IEEE Int. Conf. Perv. Comp. and Comm., White Plains, NY, U.S.A., Mar. 2007.

[14] — , "Dynamic clustering for tracking multiple transceiver-free objects," in Proc. IEEE Int. Conf. Perv. Comp. and Comm., Galveston, TX, U.S.A., Mar. 2009.

[15] D. Zhang, Y. Liu, and L. Ni, "RASS: A real-time, accurate and scalable system for tracking transceiver-free objects," in Proc. IEEE Int. Conf. Perv. Comp. and Comm., Seattle, U.S.A., Mar. 2011.

[16] D. Schuhmacher, B.-T. Vo, and B.-N. Vo, "A consistent metric for performance evaluation of multi-object filters," IEEE Trans. Signal Processing, vol. 56, no. 8, pp. 3447-3457, Aug. 2008. 